\title{
Integration of social media with healthcare big data for improved service delivery
}

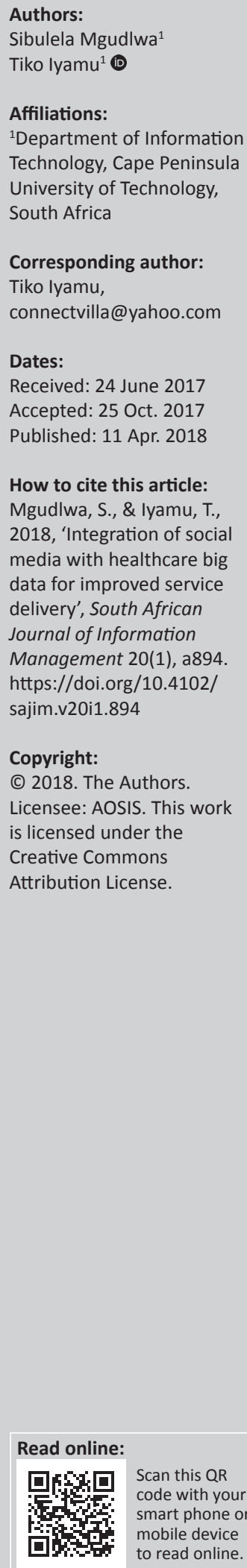

Background: In the last decade, social media users across the world have crossed 1 billion, making it one of the fastest growing sources of big data. Also, people needing healthcare continue to increase in every society. Through accessibility, communication and interaction between health practitioners and patients, this type of ever-growing, social media subscriber-based platform can be of significant use in improving healthcare delivery to society. However, users encounter serious challenges in their attempts to make use of social media and big data for health-related services. The challenges are primarily caused by factors such as integration, complexity, security and privacy. The challenges are mainly owing to the sensitive nature of the healthcare environment, as a result of personalisation and privacy of information.

Objectives: The objectives of the study were to examine and gain a better understanding of the complexities that are associated with the use of social media and healthcare big data, through influencing factors, and to develop a framework that can be used to improve health-related services to the patients.

Methods: The interpretivist approach was employed, within which qualitative data were collected. This included documents and existing literature in the areas of social media and healthcare big data. To have a good spread of both previous and current state of events within the phenomena being studied, literature published between 2006 and 2016 were gathered. The data were interpretively analysed.

Results: Based on the analysis of the data, factors of influence were found, which were used to develop a model. The model illustrates how the factors of influence can enable and at the same time constrain the use of social media for healthcare services. The factors were interpreted from which a framework was developed. The framework is intended to guide integration of social media with healthcare big data through which service delivery to patients can be improved.

Conclusion: This study can be used to guide integration of social media with healthcare big data by health facilities in the communities. The study contributes to healthcare workers' awareness on how social media can possibly be used to improve the services that they provide to the needy. Also, the study will benefit information systems and technologies and academic domains, particularly from the health services' perspective.

\section{Introduction}

The term big data is used to describe massive data sets, which are large and have more varied and complex structure with difficulties in storing, analysing and visualising for further processes or results (Sagiroglu \& Sinanc 2013). Thus, the characteristics of big data include size, velocity and variety, which are significant to organisations and businesses for various reasons (Russom 2011). These characteristics differentiate big data from normal data (Katal, Wazid \& Goudar 2013).

In recent years, the interest of individuals and organisations in big data has grown rapidly owing to the premise of its benefits. The interest spans across sectors, professions and fields of studies, such as healthcare (Kitchin 2014; Wang et al. 2015). Even though there are benefits in big data, there are numerous challenges as well. For example, the larger the size of data, the more complex it becomes in processing. As a result of these attributes and factors, Syed, Gillela and Venugopal (2013) suggested that the size of big data makes it complicated to process using on-hand database management tools or traditional data processing applications, as it often exceeds the capacity of conventional database systems. This is one of the challenges that have been experienced with social media over the years (Schroeder 2014). 
Social media produces a huge amount of data, with over a billion users worldwide, because of its rapidly increasing audience and subscribers (Carr \& Hayes 2015). The size and velocity of social media big data are based on the fact that data sets are generated in minutes and on a daily basis, from various social network platforms such as Twitter and Facebook, which have no limitations for users (Young 2014). To this end, there are claims by authors, such as Romero et al. (2011), that the growth of social media has provided millions of users with the opportunity to create and share content on a larger scale than has ever been possible before.

Big data is most relevant and useful to organisations such as healthcare service providers, with the use of big data and social media for health purposes growing in popularity in recent years (Mayer-Schönberger \& Cukier 2013). Thus, McCormack et al. (2014) proposed the analysis of big data for futuristic purposes and environmental trends. However, the use of social media and big data to improve healthcare delivery is challenged and hindered by factors, including integration (Hashem et al. 2015), security and privacy (Househ, Borycki \& Kushniruk 2014) and complexity of technology (Koster, Stewart \& Kolker 2016). This could be a contributing factor to why research in healthcare has not yet been covered extensively (De Choudhury et al. 2013). This factor fundamentally motivates this study, especially from its combination of social media and big data perspectives.

Thus, the research question was, how can social media be integrated with healthcare big data to improve service delivery? In answering the research question, we examined the factors that influence both social media and big data from a healthcare perspective, using the following questions to elicit and extract information from existing literature: (1) What are the factors that influence social media and big data? (2) What are the factors that influence big data in the healthcare environment? Based on these questions, literature was collected, reviewed and analysed.

The remainder of this article is organised into five main sections as follows: the first section presents a review of literature on big data and social media from healthcare viewpoint. The approach that was applied in the study is discussed in the second section. The third and fourth sections present the data analysis and findings, respectively. This includes a discussion about information technology (IT) integration of social media big data for improved healthcare service delivery. In the fifth section, the conclusion is drawn.

\section{Big data and social media in healthcare}

In many countries, the use of big data is a challenge, and as such it is often prioritised along with healthcare and other services (Kaisler et al. 2013). This brings different kinds of challenges to all that are involved and concerned with big data, irrespective of the level and field of work. This is one of the motivating factors behind why big data research is bound to become more widespread, and require more awareness on the part of data scientists and policymakers (Schroeder 2014) in many areas, including social media and the health sector.

Social media is a product of, as well as facilitated by, IT. Within the context of IT applications, Sagiroglu and Sinanc (2013) suggest that the use of information from big data has been very useful to companies or organisations, particularly for gaining richer and deeper insights. This suggestion has been particularly true in the areas of health and medicine, through which big data and social media activities can be remotely monitored and controlled, to manage risk behaviours and disease outbreaks (Young 2014). This is critically important in that social media is a platform that provide mechanisms for users to connect, communicate and interact with each other through which big data is generated (Correa, Hinsley \& De Zuniga 2010). Thus, social media platforms are an integral part of big data as a result of their high volume of user engagements, which produce over 500 million tweets per day on Twitter alone (Young 2014).

Primarily, users make use of social media for interaction and information sharing without geographical boundaries and with little or no limitation. Social media was, therefore, described by Carr and Hayes (2015) as internet-based channels that allow users to interact. Big data presents many benefits to businesses; however, new challenges are being presented by its existence, and these challenges are concerned with how information is stored, shared and managed (Kwon, Lee \& Shin 2014). Some of the challenges affect both the end users and the technologies that host the social media. It is within this context that Yin et al. (2012) suggested that social media brings about difficulties when it comes to sifting information that is relevant, which is sometimes as a result of its massive volumes.

Another major challenge with big data is dissemination, which could be attributed to both hardware and software, owing to size and speed of the data sets (Kaisler et al. 2013). In addition, Marx (2013) suggested that big data challenges also come from the need to engineer tools for stability and longevity, in that many software tools cannot handle the challenge and they crash often. For such reasons, big data implementations need to be analysed and executed as accurately as possible (Sagiroglu \& Sinanc 2013) in areas such as the health sector.

Big data that is generated by social media holds numerous benefits for organisations, the healthcare sector in particular. However, the use of social media in the healthcare environment poses some challenges, which include threat to privacy and the need to continuously monitor and manage where and when possible (Katal et al. 2013). The emergent threats to user online privacy comes from other users' social media, which makes this threat particularly bad, as the victims are often not involved in the process of uploading the content and as a result cannot take any pre-emptive cautions, in that the amount of data being uploaded varies so much that it cannot be detected manually (Smith et al. 2012). 


\section{Research approach}

The objective of this study was primarily to gain a better understanding of the complexities that are involved in an attempt to integrate social media with healthcare big data, for improved services. Based on this objective, the interpretivist approach was selected within which the qualitative methods were employed. The interpretivist approach was applied mainly because it focuses on social interactions, including the meanings that human beings associate with various contexts and their relations with each other (Tekin \& Kotaman 2013).

Qualitative data were collected based on the objective purposely to enhance the quality and richness of the data (Creswell \& Poth 2017). Through the qualitative methods, rich and nuanced data can be gathered, even from complex phenomena (Coast et al. 2012). Also, this was primarily because the qualitative methods richly concern direct and necessary materials that relate to the phenomena being studied (Kleinsmann, Buijs \& Valkenburg 2010). Thus, from the qualitative perspective, existing literature were gathered and used as data in this study. According to Iyamu, NehemiaMaletzky and Shaanika (2016:171), the purpose of the use of literature as data is to have a spread of historical perspectives in terms of the consistency of the meaning that has been associated with the concepts, as well as the challenges that exist over a period of time. The areas of focus in the collection of data were big data, healthcare, social media and IT integration, as shown in Table 1.

In gathering the literature, two main search engines were used, Google Scholar (https://scholar.google.co.za) and the databases in the system of Cape Peninsula University of Technology (CPUT) (http:/ / www.cput.ac.za/lib/databases). Three of the 101 databases in CPUT'S system were used as sources for literature gathering. The databases include ProQuest, SAGE publications and EBSCOhost. The databases were selected primarily because of their focus on information systems and technologies (IS/IT), health sciences and social sciences, which cover social media, healthcare, IS/IT and big data. Three criteria were used, they include areas of study (social media and healthcare), keywords (such as big data, integration, data security and data privacy), and year range (2006-2016) of publication.

As shown in Table 1, peer-reviewed literature between the years 2006 and 2016 were collected within the areas of focus and used as data. This was to have a good spread of the previous and current state of events within the phenomena being studied. As presented in Table 1, 14 peer-reviewed articles were gathered. The quality rather than the size was more important to this, a qualitative study, as explained by Cleary, Horsfall and Hayter (2014). While focusing on the phenomena, it ensured that the literature covered both developing and developed countries. This helped to balance the activities as they unfold in both worlds but from different perspectives. The interpretive approach was selected for the analysis of the data, because it helped to examine and gain an understanding of social reality (Choy 2014). Also, the interpretive approach guides rigour and leads the researcher to the depth of the data (Venkatesh, Brown \& Bala 2013).

The interpretive approach was used in this study in order to examine and interpret factors and trends as they manifests in the integration of IS/IT with other components that are associated with healthcare services, from the social media and big data standpoints. The interpretive approach can be defined as a lens through which the choice of qualitative methods can be influenced (Goldkuhl 2012). Also, the interpretive approach

TABLE 1: Existing literature as data.

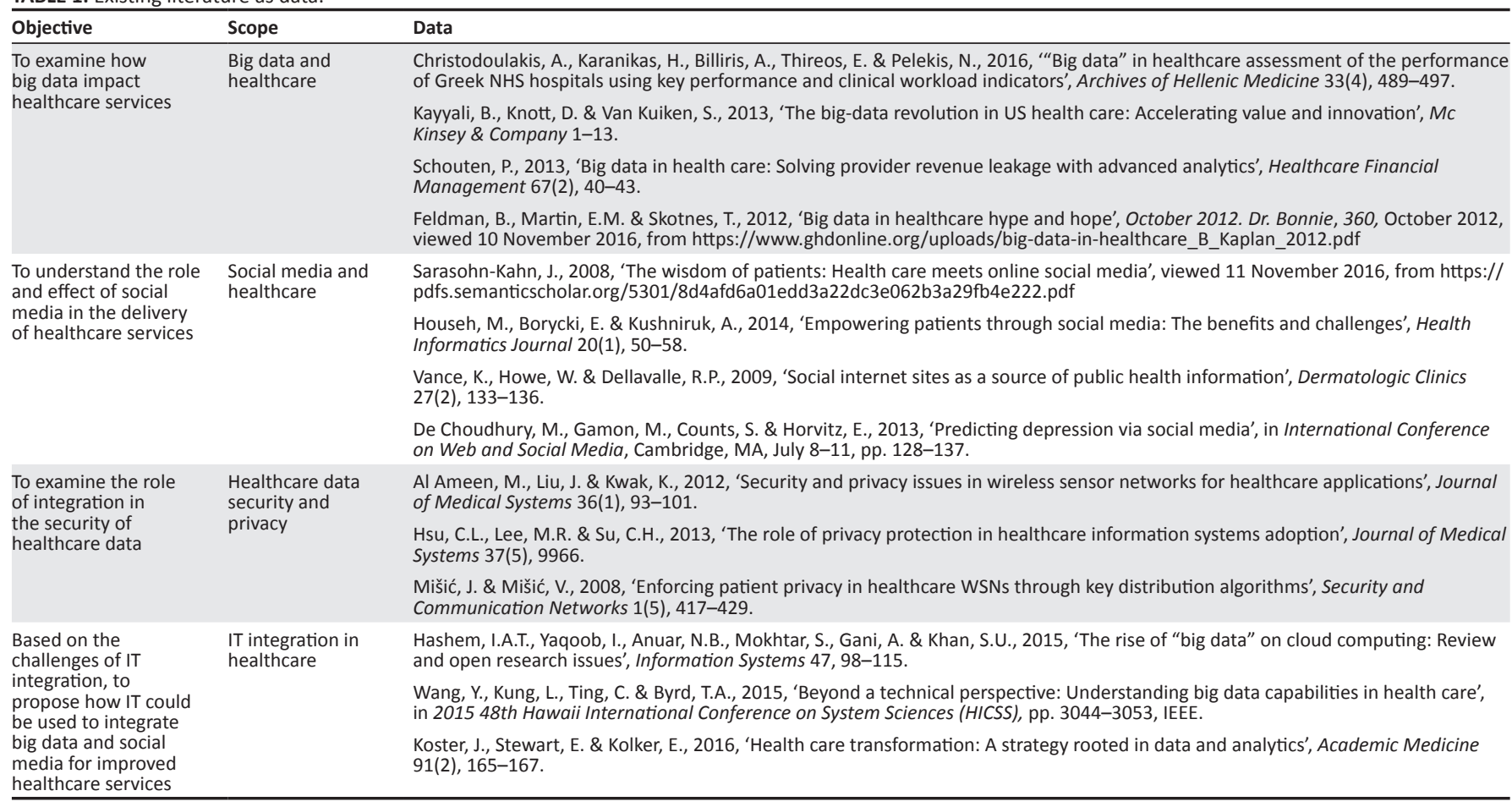


is considered to be a realist ontology, which stands to argue that all observation consists of theory and value, and that investigating the social world is not to seek detachment from objective truth. Most importantly, the interpretive approach focuses on culturally derived and historically situated interpretations of the social world (Gray 2013).

\section{Analysis and discussion}

The analysis of the data was conducted based on the following research questions: (1) What are the factors that influence social media and big data? and (2) What are the factors that influence big data in healthcare? Factors from both questions were combined, as shown in Figure 1. The relationship that exists between the factors and how they are interconnected to influence improved healthcare services using social media big data is illustrated in Figure 1.

\section{What are the factors that influence social media big data?}

Social media provides benefits as well as challenges to both users and host providers through which big data is generated or connected. Even though challenges exist, there is a growing trend in the use of social media technology by patients to seek and search for health information (Househ et al. 2014). It was thus highlighted as fact by Vance, Howe and Dellavalle (2009) that social media platforms such as Facebook, MySpace and Twitter are rapidly increasing as sources of health big data.

The benefits as well as challenges come from both technical and non-technical perspectives. Within these challenges, De Choudhury et al. (2013) examined the potential of social media as a tool in detecting and predicting affective disorders in individuals. Thus, within the context of healthcare, some of the factors that influence social media and big data include (1) lack of limitation to accumulation of big data; (2) ease of connectivity among subscribers; (3) lack of geographical boundaries; and (4) real-time access to big data. These factors are discussed as follows:

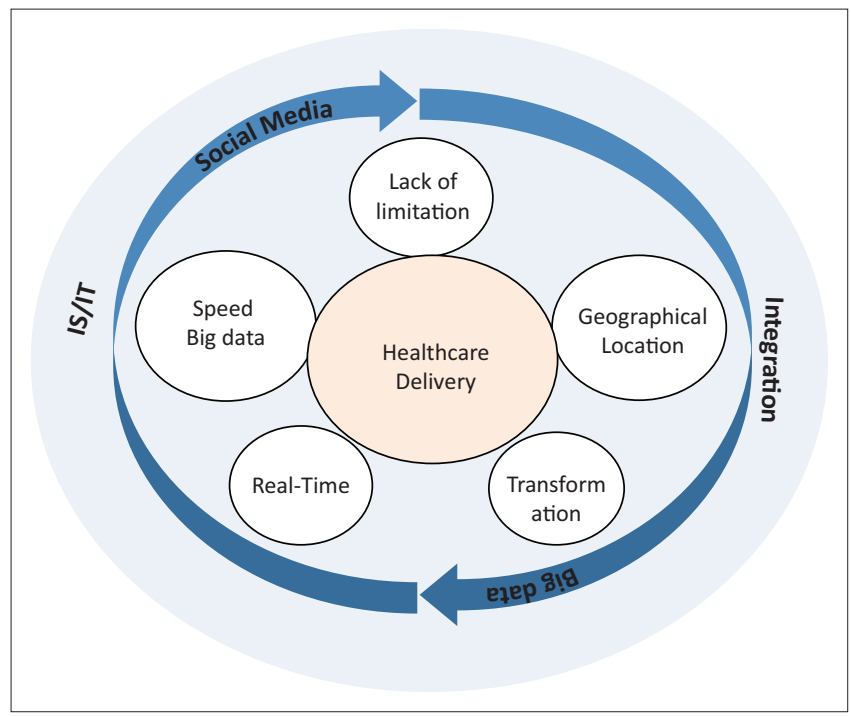

FIGURE 1: Factors of influence for social media and healthcare big data.
- Lack of limitation - There is no limit to the amount of data that are accumulated and shared between social media users. However, the infrastructure used to store the big data is sometimes challenged, either from the users or host's perspective, or both. There is a constant flow of big data, which is accumulated at an unprecedented rate and presents new challenges. These challenges are owing to the rapidity of volume and variety of big data that are collected, which ultimately change in their velocity at different rates, which influences decision-making (Feldman, Martin \& Skotnes 2012:10). Depending on the capacity of the hardware, both users and host providers do sometimes run out of storage space (Hashem et al. 2015). Also, the amount of data that is generated from activities that are facilitated by the Internet and mobile technologies is unprecedented (Vayena et al. 2015). However, this challenge seems to be easier to manage by both users and those hosting the infrastructures (Wang et al. 2015), as they filter the types of data sets that are stored.

- Ease of connectivity - Social media platforms have facilitated person-to-person, person-to-business and business-to-business connections. The connectivity has increased visibility, which manifests to add value for some of the users. From a technical perspective, connectivity through social media is effortless in that the infrastructures are scalable and reused (Christodoulakis et al. 2016). Various technologies and devices, such as database technologies and patient monitoring and sensor technologies, are used to generate health big data by hospitals and medical organisations at an unusual speed. One of the premises of social media in healthcare is its support and enablement of patient-to-patient, patientto-facility and facility-to-facility engagement (Househ et al. 2014). Sarasohn-Kahn (2008) suggested that social media convergence enables personalisation in terms of messaging and model of delivery. Personalisation is a challenge that will always exist within healthcare big data. Thus, Kayyali, Knott and Van Kuiken (2013) argued that privacy concerns will continue to be problematic in the use of big data for healthcare services.

- Real-time - Another important benefit of social media is its ability to allow real-time big data capture and accessibility. In some medical situations, access to realtime big data, such as trauma monitoring for blood pressure and bedside heart monitors, are essential to life or death (Feldman et al. 2012). Real-time access to health activities is critical particularly in monitoring to give immediate alerts of changes in patient status and to correlate with patient records (Al Ameen, Liu \& Kwak 2012). Also, real-time access requires compatibility with various devices, allowing usage, accessibility and management of social media and big data at any time and from any geographical location. As explained by Kayyali et al. (2013), irrespective of the challenges, integration of social media with big data by IS/IT allows medical personnel and health facilities to deliver evidence-based care that is more coordinated and personalised. This has potential to improve performance 
in areas such as prevention and care coordination. In situations where patients' data may be collected through automated systems, and in real time, there is a high possibility of constraints (Mišić \& Mišić 2008). Thus, software applications and other devices that are used to store, access and manage health data should be integrated with mechanisms such as privacy protection, access control and secure transmission modules (Hsu, Lee \& Su 2013).

- Speed of social media and big data - Social media enables speedy management of patients' chronic medications through its integration with IS/IT, which allows monitoring (Sarasohn-Kahn 2008). For example, through integration of big data into legacy IT systems, health practitioners can automatically monitor drug safety by tracking warning signals that are triggered by alarm systems (Wang et al. 2015). Thus, Kayyali et al. (2013) argued that the integration of IS/IT solution, such as HealthConnect, with big data improves outcomes in cardiovascular disease and promotes the use of electronic health records for data sharing and exchange across medical facilities. The use of social media increases interaction and dissemination of health information among community members and improves health services (Vance et al. 2009). This contributes to the increasing interest in the integration of social media with healthcare big data. For example, through social media, De Choudhury et al. (2013) employed big data to learn more about people's social and psychological behaviour in order to predict their vulnerabilities to depression.

\section{What are the factors that influence big data in healthcare?}

Obviously, the communications and interactions between patients and medical practitioners through technological devices are mostly wireless, which result in various security threats and challenges to personal information ( $\mathrm{Al}$ Ameen et al. 2012). Primarily, the factors that can influence big data within the healthcare environment include (1) lack of geographical boundaries, (2) transformation and (3) security and privacy, discussed in detail below:

- Lack of geographical boundaries - IS/IT artefacts are used to facilitate activities towards improving the efficiency and quality of healthcare services (Hsu et al. 2013). Users are not restricted to their geographical locations in the application of social media. One of the reasons is the integration of technologies, such as cloud computing, that are used to perform massive-scale, complex and sensitive tasks, which include big data (Hashem et al. 2015). This makes the use and dissemination of big data more purposeful. Also, the lack of geographical boundaries increases communication between medical practitioners and patients that are far apart. This eradicates duplication and replication of infrastructures. Technological devices can be used to collect, access and manage big data, remotely and from different locations
(Al Ameen et al. 2012). However, the host databases should be situated in a secured location to prevent theft and destruction (Mišić \& Mišić 2008).

- Transformation - Rapidly increasing patients' heterogeneous big data helps to provide a valuable resource for use in the course of improving healthcare service delivery, which include medical decision-making (Christodoulakis et al. 2016). In recent years, there has been optimism in the use of big data to transform healthcare services, but some structural issues continue to be obstacles (Kayyali et al. 2013). However, transformation will be difficult because of manipulation of identity and misinformation about patients' records, which are of privacy and security concerns (Househ et al. 2014). Healthcare transformation can gain from the integration of IS/IT solutions with big data (Wang et al. 2015). Innovation such as digitisation is instrumental to accelerating transformation of healthcare services (Koster et al. 2016). Integration of social media and big data helps to eliminate the need to maintain expensive computing hardware, dedicated storage space and software applications (Hashem et al. 2015). Thus, integration of IS/ IT capabilities is key to healthcare transformation (Wang et al. 2015) for improved services.

- Security and privacy - This poses challenges to both the users and technology host providers. The users have to continuously be wary about the privacy of their personal information. The technology provider manages the volume of big data, organises its variety and responds to the rapid speed (velocity) at which they receive, process and disseminate the big data. Feldman et al. (2012) argued that privacy of patients' big data has increasingly become a matter of attention and concern as social media exposes personal data to potential misuse and mismanagement. Even though Kayyali et al. (2013) highlighted some benefits of healthcare big data, such as enabling faster identification of high-risk patients, more effective interventions and closer monitoring, there are security challenges. The challenges emanate from evidence relating to how the efficacy and effectiveness of big data from social media are currently limited owing to security and privacy concerns (Househ et al. 2014). The main challenges are security and privacy.

\section{Findings and discussion: Information technology integration of social media and big data for healthcare services}

From the analysis of the data, using the interpretive approach, four factors were found to be critical in achieving the objectives of the study. As shown in Figure 2, the four factors must be tightly connected in order to achieve improved healthcare service delivery from the use of social media and big data through integration of IS/IT artefacts. The factors include (1) analytics of big data; (2) the roles and influence of data from social media; (3) integration of big data with social 


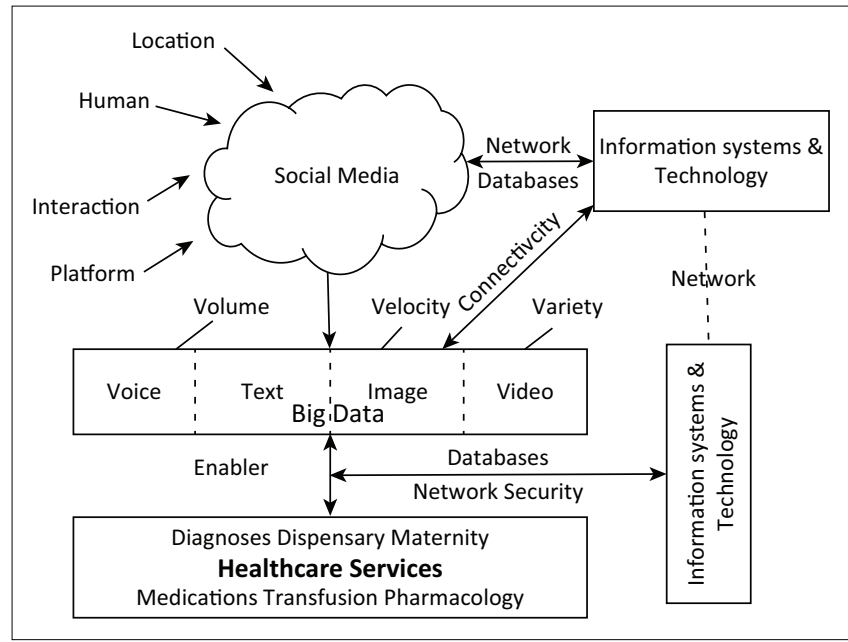

FIGURE 2: Information technology integration of social media with healthcare big data.

media, using IS/IT artefacts; and (4) healthcare and privacy within the context of big data that comes from social media.

\section{Big data and analytics}

The continuous increase in the speed and variety of big data makes it necessary for analysis to be constantly conducted before the data sets are accessed and used to ensure accuracy and appropriateness. Regular analysis of the healthcare big data of each patient is important owing to the numerous activities that are carried out on the patients, using various apparatus and diagnostic tools (Christodoulakis et al. 2016). Thus, different analytics tools have been introduced and applied over the years. However, it has been noted that some analytics tools are problematic owing to reasons such as lack of capability for use in real time, lack of sensitivity and slow response time. This has contributed to making big data and data analytics tools slow or disruptive to innovations, primarily because they are difficult to integrate with each other, in Kitchin's (2014) assessment.

Also, continuous change in the size and variety of big data is a challenge that affects how the data sets are stored, processed accessed and transmitted, which ultimately influences the analysis. The constant increases in the volume, variety and speed of big data make it even more complex and difficult for healthcare organisations to achieve their objectives (Sagiroglu \& Sinanc 2013). The complexities and challenges have impact on big data at various levels of security and privacy; hence, there is a need to conduct regular analysis. Integration of the tools and artefacts that are involved in the collection, storage and analytics is therefore critical. This is primarily to reduce complexity and enhance the capacity to enable and support healthcare activities for improved services to society.

Thus, there is a need for analytics tools that can be integrated with the enabling and associated artefacts, such as operating systems, databases and healthcare applications. The integration helps flexibility and compatibility between social media big data analytics tools and other IS/IT supporting and enabling artefacts within the context of healthcare services. As the data increase in size and complexity, the integration maintains and enhances the connectivity and relationship between big data analytics tools and social media platforms for the purposes of improving healthcare services to the communities.

\section{Social media and information system and/or information technology}

The increasing volume and expected speed of users' data sets are caused and influenced by various factors, which include database and network capacity. This is an indication that social media and healthcare big data can be queried or requested on a real-time basis. However, for epidemiological and biomedical data, there is often a delay in time between when people contract a virus or disease and the release of the data (Young 2014). This could be associated with the different systems and technologies that are used to host, connect and transmit big data for various usefulness.

The use of social media continues to vastly expand the reach of the Internet network and other IS/IT associated components. IS/IT provide systems that are used for accessing and interacting with social media networks, which comprises of hosting, processing and transmission devices. One of the challenges is that some of these devices are not, or cannot be, integrated. Hence some social media platforms, such as YouTube, Facebook and Twitter, are too often treated as stand-alone elements rather than part of an integrated system for health-related processes and activities (Hanna, Rohm \& Crittenden 2011). The integrations of these systems and devices are significant to how they respond and the result that they reproduce for service delivery.

Over the years, an increasing number of patients and healthcare service providers have embraced the use of social media for health-related activities and processes. Even though subscribers and medical practitioners make use of social media for the same health-related activities, the motives or rationale can be, and often are, different. However, little is known yet about the motives behind patients' and health professionals' use of social media for health-related reasons (Antheunis, Tates \& Nieboer 2013). This necessitates a tighter security and privacy measure of social media big data for health-related activities by both patients and health professionals.

\section{Healthcare and privacy}

Health-related issues are at all times considered to be private because of their nature of sensitivity and care. This is primarily to protect both the service providers and those requiring the services. As a result, many healthcare service providers have policies on code of conduct, which are intended to guide the privacy of their activities. However, the challenges persist. This could be attributed to the fact that research about healthcare information systems (HISs) pays little attention to privacy protection (Hsu et al. 2013). 
A common understanding is that privacy of patients' big data is a foremost security concern in providing healthcare services and must be enforced through the use of strong technology, such as cryptography, irrespective of the source of data (Mišić \& Mišić 2008). In search of solutions, Hsu et al. (2013) proposed a framework that could be used to protect the electronic healthcare records (EHRs) of patients.

Consequently, big data sets that generate diagnoses, medications and other health-related activities for care must be secured and adhere to the privacy policies. This is supported and enabled by the software and hardware that hosts and facilitates the use of social media with healthcare big data. Therefore, big data that are generated from social media activities require a security-integrated approach with the enabling and supporting IT artefacts, which include network, Internet and databases. This is primarily to address security and privacy concerns, to which $\mathrm{Al}$ Ameen et al. (2012) suggested that there should be more focus on wireless sensor networks and applications. This approach will assist in the control and management of flows and accessibility of big data sets in their increasing volume, speed and variety.

\section{Information system and/or information technology and integration}

The flow of materials, products and services in both shortterm and long-term operations are facilitated, enabled and supported by IS/IT artefacts such as software, hardware and networks. These flows are engineered through linkage between the artefacts of IS/IT, which include the cloud and databases. None of the artefacts have the capacity to execute operations in isolation. Thus, integration with IS/IT constitutes interrelations and connectivity among its artefacts.

In order to make use of big data that comes from social media for healthcare services, IS/IT artefacts, which are associated with various entities such as the cloud, network and data, must be integrated. On the one hand, healthcare big data are hosted by the necessary software and hardware, which are interconnected and interrelated. On the other hand, the existence of social media is enabled and powered by network connectivity. There is a relationship between big data and the network in every operation of social media and healthcare that reproduces big data.

\section{Conclusion}

This article is intended to be of benefit to both the healthcare and academic domains, from theoretical, methodological and practical perspectives. Theoretically, this study reveals the factors that can influence the use of social media big data for improved healthcare, which both organisations and academics will find useful in their pursuit for advancement. In addition, the use of social media big data as a form of research has become a trend, which ultimately draws the attention of both the business and healthcare sectors. Another theoretically important contribution is that this article adds to existing literature in the areas of big data, healthcare and social media. Methodologically, the integration of the three areas as highlighted by the study contributes to both organisations and academics through its approach towards real circumstances that were revealed. This includes how social media-generated big data can be used to improve health-related services, which include remote monitoring and surveillance of risk behaviours and disease outbreaks. A practical contribution of the study is in its insights, which managers and academia can consider in order to gain better understanding of the influencing factors towards managing, storing and retrieving social media big data and using IS/IT to transform them for improved healthcare services to society.

Having identified the primary areas of concern as well as benefits in the intersection between social media, healthcare and big data, it is essential to consider ethical issues. The privacy of patient big data has to be prioritised and should be guided by the ethical considerations of communities. This is one of the areas for future study as research in healthcare and social media big data continue to evolve, particularly in developing countries.

\section{Acknowledgements Competing interests}

The authors declare that they have no financial or personal relationships which may have inappropriately influenced them in writing this article.

\section{Authors' contributions}

S.M. and T.I. defined the scope and design of the research. S.M. was responsible for the data collection. The analysis of the data was led by T.I. Both authors carried out the write-up of the article.

\section{References}

Al Ameen, M., Liu, J. \& Kwak, K., 2012, 'Security and privacy issues in wireless sensor networks for healthcare applications', Journal of Medical Systems 36(1), 93-101. https://doi.org/10.1007/s10916-010-9449-4

Antheunis, M.L., Tates, K. \& Nieboer, T.E., 2013, 'Patients' and health professionals' use of social media in health care: Motives, barriers and expectations', Patient Education and Counseling 92(3), 426-431. https://doi.org/10.1016/j.pec.2013. 06.020

Carr, C.T. \& Hayes, R.A., 2015, 'Social media: Defining, developing, and divining', Atlantic Journal of Communication 23(1), 46-65. https://doi.org/10.1080/154568 70.2015 .972282

Choy, L.T., 2014, 'The strengths and weaknesses of research methodology: Comparison and complimentary between qualitative and quantitative approaches' IOSR Journal of Humanities and Social Science 19(4), 99-104. https://doi.org/ IOSR Journal of Humanities
10.9790/0837-194399104

Christodoulakis, A., Karanikas, H., Billiris, A., Thireos, E. \& Pelekis, N., 2016, “"Big data” in health care', Archives of Hellenic Medicine/Arheia Ellenikes latrikes 33(4), 489497.

Cleary, M., Horsfall, J. \& Hayter, M., 2014, 'Data collection and sampling in qualitative research: Does size matter?', Journal of Advanced Nursing 70(3), 473-475. https://doi.org/10.1111/jan.12163

Coast, J., Al-Janabi, H., Sutton, E.J., Horrocks, S.A., Vosper, A.J., Swancutt, D.R. et al., 2012, 'Using qualitative methods for attribute development for discrete choice experiments: Issues and recommendations', Health Economics 21(6), 730-741. https://doi.org/10.1002/hec.1739

Correa, T., Hinsley, A.W. \& De Zuniga, H.G., 2010, 'Who interacts on the web?: The intersection of users' personality and social media use', Computers in Human Behaviour 26(2), 247-253. https://doi.org/10.1016/j.chb.2009.09.003

Creswell, J.W. \& Poth, C.N., 2017, Qualitative inquiry and research design: Choosing among five approaches, Sage, London, UK. 
De Choudhury, M., Gamon, M., Counts, S. \& Horvitz, E., 2013, 'Predicting depression via social media', in International Conference on Web and Social Media, Cambridge, MA, July 8-11, pp. 128-137.

Feldman, B., Martin, E.M. \& Skotnes, T., 2012, 'Big data in healthcare hype and hope', Dr. Bonnie 360, October 2012, viewed 10 November 2016, from https://www. ghdonline.org/uploads/big-data-in-healthcare_B_Kaplan_2012.pdf

Goldkuhl, G., 2012, 'Pragmatism vs interpretivism in qualitative information systems research', European Journal of Information Systems 21(2), 135-146. https://doi. org/10.1057/ejis.2011.54

Gray, D.E., 2013, Doing research in the real world, Sage, Los Angeles, CA.

Hanna, R., Rohm, A. \& Crittenden, V.L., 2011, 'We're all connected: The power of the social media ecosystem', Business Horizons 54(3), 265-273. https://doi. org/10.1016/j.bushor.2011.01.007

Hashem, I.A.T., Yaqoob, I., Anuar, N.B., Mokhtar, S., Gani, A. \& Khan, S.U., 2015, 'The rise of "big data" on cloud computing: Review and open research issues', Information Systems 47, 98-115. https://doi.org/10.1016/j.is.2014.07.006

Househ, M., Borycki, E. \& Kushniruk, A., 2014, 'Empowering patients through social media: The benefits and challenges', Health Informatics Journal 20(1), 50-58. https://doi.org/10.1177/1460458213476969

Hsu, C.L., Lee, M.R. \& Su, C.H., 2013, 'The role of privacy protection in healthcare information systems adoption', Journal of Medical Systems 37(5), 1-12. https:// doi.org/10.1007/s10916-013-9966-z

Iyamu, T., Nehemia-Maletzky, M. \& Shaanika, I., 2016, 'The overlapping nature of business analysis and business architecture: What we need to know', The Electronic Journal Information Systems Evaluation 19(3), 169-179.

Kaisler, S., Armour, F., Espinosa, J.A. \& Money, W., 2013, 'Big data: Issues and challenges moving forward', in 46th Hawaii International Conference on System Sciences (HICSS), IEEE, Wailea, Maui, HI, January 7-10, pp. 995-1004.

Katal, A., Wazid, M. \& Goudar, R.H., 2013, 'Big data: Issues, challenges, tools and good practices', in 2013 Sixth International Conference on Contemporary Computing (IC3), IEEE, Noida, India, August 8-10, pp. 404-409.

Kayyali, B., Knott, D. \& Van Kuiken, S., 2013, 'The big-data revolution in US health care: Accelerating value and innovation', Mc Kinsey \& Company 2(8), 1-13.

Kitchin, R., 2014, 'Big data, new epistemologies and paradigm shifts', Big Data \& Society 1(1), 1-12.

Kleinsmann, M., Buijs, J. \& Valkenburg, R., 2010, 'Understanding the complexity of knowledge integration in collaborative new product development teams: A case study', Journal of Engineering and Technology Management 27(1), 20-32. https:// doi.org/10.1016/j.jengtecman.2010.03.003

Koster, J., Stewart, E. \& Kolker, E., 2016, 'Health care transformation: A strategy rooted in data and analytics', Academic Medicine 91(2), 165-167. https://doi.org/ 10.1097/ACM.0000000000001047

Kwon, O., Lee, N. \& Shin, B., 2014, 'Data quality management, data usage experience and acquisition intention of big data analytics', International Journal of Information Management 34(3), 387-394. https://doi.org/10.1016/j.ijinfomgt.2014.02.002

Marx, V., 2013, 'Biology: The big challenges of big data', Nature 498(7453), 255-260. https://doi.org/10.1038/498255a

Mayer-Schönberger, V. \& Cukier, K., 2013, Big data: A revolution that will transform how we live, work, and think, Hachette, London, UK.
McCormack, L.A., Friedrich, C., Fahrenwald, N. \& Specker, B., 2014, 'Feasibility and acceptability of alternate methods of postnatal data collection', Maternal and Child Health Journal 18(4), 852-857. https://doi.org/10.1007/s10995-013-1310-1

Mišić, J. \& Mišić, V., 2008, 'Enforcing patient privacy in healthcare WSNs through key distribution algorithms', Security and Communication Networks 1(5), 417-429. https://doi.org/10.1002/sec.40

Romero, D.M., Galuba, W., Asur, S. \& Huberman, B.A., 2011, 'Influence and passivity in social media', in Proceedings of the 20th International Conference Companio on World Wide Web, ACM, Hyderabad, India, March 28-April 01, pp. 113-114.

Russom, P., 2011, Big data analytics, TDWI best practices report, fourth quarter, pp. 19,40 , viewed 11 November 2016, from http://tdwi.org/research/2011/09/best practices-report-q4-big-data-analytics.aspx

Sagiroglu, S. \& Sinanc, D., 2013, 'Big data: A review', in 2013 International Conference on Collaboration Technologies and Systems (CTS), IEEE, San Diego, CA, May 20-24, pp. 42-47.

Sarasohn-Kahn, J., 2008, 'The wisdom of patients: Health care meets online social media', viewed 11 November 2016, from https://pdfs.semanticscholar.org/5301/ 8d4afd6a01edd3a22dc3e062b3a29fb4e222.pdf

Schouten, P., 2013, 'Big data in health care: Solving provider revenue leakage with advanced analytics', Healthcare Financial Management 67(2), 40-43.

Schroeder, R., 2014, 'Big data and the brave new world of social media research', Big Data \& Society 1(2), 1-11.

Smith, M., Szongott, C., Henne, B. \& Von Voigt, G., 2012, 'Big data privacy issues in public social media', in 2012 6th IEEE International Conference on Digital Ecosystems Technologies (DEST), IEEE, Campione d'Italia, Italy, June 18-20, pp. 1-6.

Syed, A., Gillela, K. \& Venugopal, C., 2013, 'The future revolution on big data', International Journal of Advanced Research in Computer and Communication Engineering 2(6), 2446-2451.

Tekin, A.K. \& Kotaman, H., 2013, 'The epistemological perspectives on action research', Journal of Educational and Social Research 3(1), 81-91.

Vance, K., Howe, W. \& Dellavalle, R.P., 2009, 'Social internet sites as a source of public health information', Dermatologic Clinics 27(2), 133-136. https://doi.org/ 10.1016/j.det.2008.11.010

Vayena, E., Salathé, M., Madoff, L.C. \& Brownstein, J.S., 2015, 'Ethical challenges of big data in public health', PLOS Computational Biology 11(2), 1-7. https://doi. org/10.1371/journal.pcbi.1003904

Venkatesh, V., Brown, S.A. \& Bala, H., 2013, 'Bridging the qualitative-quantitative divide: Guidelines for conducting mixed methods research in information systems', MIS Quarterly 37(1), 21-54. https://doi.org/10.25300/MISQ/2013/37. 1.02

Wang, Y., Kung, L., Ting, C. \& Byrd, T.A., 2015, 'Beyond a technical perspective: Understanding big data capabilities in health care', in 48th Hawaii International Conference on System Sciences (HICSS), IEEE, Grand Hyatt, HI, January 5-8, pp. 3044-3053.

Yin, J., Lampert, A., Cameron, M., Robinson, B. \& Power, R., 2012, 'Using social media to enhance emergency situation awareness', IEEE Intelligent Systems 27(6), 52-59. https://doi.org/10.1109/MIS.2012.6

Young, S.D., 2014, 'Behavioural insights on big data: Using social media for predicting biomedical outcomes', Trends in Microbiology 22(11), 601-602. https://doi. org/10.1016/j.tim.2014.08.004 